\title{
MORSE AND GENERIC CONTACT BETWEEN FOLIATIONS
}

\author{
BY
}

\author{
RUSSELL B. WALKER
}

\begin{abstract}
Motivated by the recent work of J. Franks and C. Robinson, the study of the contact between two foliations of equal codimension is begun. Two foliations generically contact each other in certain dimensional submanifold complexes. All but a finite number of these contact points are "Morse". In a recent paper by the author, a complete large isotopy "index of contact" is specified for two foliations of $T^{2}$. If contact is restricted to index 0 ("domed contact"), some sharp conclusions are made as to the topology of the manifold and isotopy classes of the two foliations. It is hoped that this work will lead to the construction of new quasi-Anosov diffeomorphisms and possibly to a new Anosov diffeomorphism.
\end{abstract}

\section{Preliminaries.}

Introduction. In $\S 1$, contact points (i.e., the points where the two foliations are not transverse) are shown to be either nice (Morse) or not so nice (non-Morse). A generalized "Lemma of Morse" is proved. Then the generic situation (isotopy topology) is worked out in Theorem 1. For example, two codimension 1 foliations of a compact manifold generically have contact, the finite union of circles; furthermore, all but a finite number of these contact points are Morse. The proof is complicated by the fact that the plane fields must be perturbed through integrable plane fields.

To produce a quasi-Anosov diffeomorphism which was not Anosov, John Franks and Clark Robinson [1] needed to isotope two 1-dimensional foliations of $S^{2} \times I$, nontangent (or "clean"). This isotopy had to be carefully and painstakingly constructed. An invariant of "isotransversability" is not available except on $T^{2}$ where the complete large isotopy problem is known [14]. But in higher dimensions, the situation is still overbearing.

The last section contains a sharp result about two foliations of a compact manifold with boundary where the contact is restricted to "domed" contact. Such contact can only occur between foliations of thick spheres one of which is the trivial foliation, the other an element of one of five certain isotopy classes.

I would especially like to thank my advisor, Charles Pugh, for his great help and encouragement along the way. Blaine Lawson, Glenn Davis, and James Faran for their technical advice; my parents for some fine drawings

Received by the editors September 28, 1978.

AMS (MOS) subject classifications (1970). Primary 57D30; Secondary 58F15, 58F10. 
and many bucks; and my wife, Angelyn, and son, Oscar, for so much support and encouragement over the years.

Definitions. A good reference for background material and basic definitions is Blaine Lawson's survey article [5].

A $C^{\infty}$ smooth codimension $k$ foliation $\mathscr{F}$ of a manifold $M^{n}$ is a collection of leaves $\{\mathcal{L}\}$ such that for all $x \in M$ there exists a locally defined smooth foliation chart $f: U \rightarrow \mathbf{R}^{k}, x \in U$, for which all $y \in U$ are regular points and such that $\{\mathfrak{L} \cap U: \mathcal{E} \in \mathscr{F}\}$ are the level surfaces of $f$. If $M$ is a manifold with boundary then $\mathscr{F}$ is a proper foliation of $M$ if $\mathscr{F}$ foliates int $M$ and $\mathscr{F} \cap \partial M$ is a codimension $(k-1)$ foliation. So if $k=1$, the boundary components are leaves of $\mathscr{F}$.

$\mathcal{F}$ is a foliation with boundary of $M$ if $\mathcal{F}$ may be extended to a foliation of an open neighborhood of $M$ ( $M$ thought of as being embedded in some Euclidean space).

A codimension 1 foliation $\mathscr{F}$ is transversely orientable if there exists a nonsingular vector field transverse to $\mathcal{F}$. In this paper all codimension 1 foliations will be transversely orientable. A trivial foliation of $M \times N$ has leaves of the form $M \times\{x\}, x \in N$. $U$ is a foliation neighborhood of a leaf $\mathcal{L}=M \times\left\{x_{0}\right\}$ of a trivial foliation if $U=M \times B\left(x_{0}\right)$ (an open ball about $x_{0}$ contained in $\left.N\right)$ and $U$ is foliated by $\left\{M \times\{x\}: x \in B\left(x_{0}\right)\right\}$.

Associated to a codimension $k$ foliation $\mathcal{F}$ is a tangent $k$-plane field $\sigma_{\mathscr{F}}$ These plane fields are sections of the $(n, n-k)$-Grassmannian bundle over $M$ :

$$
\begin{array}{cc}
G_{n, n-k} \quad \hookrightarrow \quad G B_{n, n-k} \\
\sigma_{\mathcal{F}} \uparrow \downarrow \pi \\
M
\end{array}
$$

Plane fields associated to foliations must satisfy the Frobenius conditions; such plane fields are called integrable. Two foliations $\mathcal{F}$ and $\mathcal{G}$ of $M$ are $C^{\infty}$-close if $\sigma_{\mathscr{F}}$ and $\sigma_{\mathcal{G}}$ are $C^{\infty}$-close. Designate by Fol $_{k}(M)$ the set of all $C^{\infty}$ smooth codimension $k$ foliations with the $C^{\infty}$-topology. $P$ is a generic property of foliations $\mathscr{F} \in \mathrm{Fol}_{k}(M)$ if $P$ holds for a residual subset of $\mathrm{Fol}_{k}(M)$.

Two foliations $\mathcal{F}$ and $\mathcal{G}$ are isotopic or $\mathcal{F} \sim \mathcal{G}$ if there exists a diffeomorphism $f$ isotopic to the identity such that $f(\mathscr{F})=\mathcal{G}$. In Lawson [6], the term completely equivalent has the same meaning. Two foliations are homotopic if their plane fields are homotopic through integrable plane fields. The homotopy class of $\mathscr{F}$ is designated by [ $\mathscr{F}]$.

For $\mathcal{F}$ and $\mathcal{G}$ two codimension $k$ foliations of $M^{n}$, let $T_{x} \mathcal{F}$ denote the tangent space to the leaf of $\mathscr{F}$ through $x$, similarly for $T_{x} \mathcal{G}$ (so $T_{x} \mathscr{F}$ and $T_{x} \mathcal{G}$ are both $(n-k)$-dimensional). For $2 k \leqslant n$, $\mathscr{F}$ and $\mathcal{G}$ are transverse, if $T_{x} \mathcal{F}+T_{x} \mathcal{G}=T_{x} M$ (at each $\left.x \in M\right)$. For unrestricted $k, \mathcal{F}$ and $\mathcal{G}$ are clean 
if $\operatorname{dim}\left(T_{x} \mathcal{F}+T_{x} \mathcal{G}\right)=\min (n, 2(n-k))$, for each $x \in M$ (so $\mathscr{F}$ and $\mathcal{G}$ are as "transverse" as possible). Transverse implies clean. The set of points of $M$ where $\mathscr{F}$ and $\mathcal{G}$ are not clean is called the contact set or $\operatorname{Con}(\mathscr{F}, \mathcal{G})$. The set of points where $\mathcal{F}$ and $\mathcal{G}$ are tangent is called the tangency set or $\operatorname{Tan}(\mathcal{F}, \mathcal{G})$. $\mathscr{F}$ and $\mathcal{G}$ are isocleanable if there exists an isotopy $h_{t}: M つ$ such that $h_{1}(\mathscr{F})$ is clean to $\mathcal{G}$. Similarly for isotransversable. A Reeb sector is a solid cylinder $D^{n-1} \times I$ which has a foliation with boundary $\mathscr{F}$ defined by $f\left(x_{1}, \ldots, x_{n}\right)=$ $\left(x_{1}^{2}+x_{2}^{2}+\cdots+x_{n-1}^{2}\right)-x_{n}$ and is such that $\partial D^{n-1} \times I$ is a leaf of $\mathscr{F}$. (See Figure 1.)

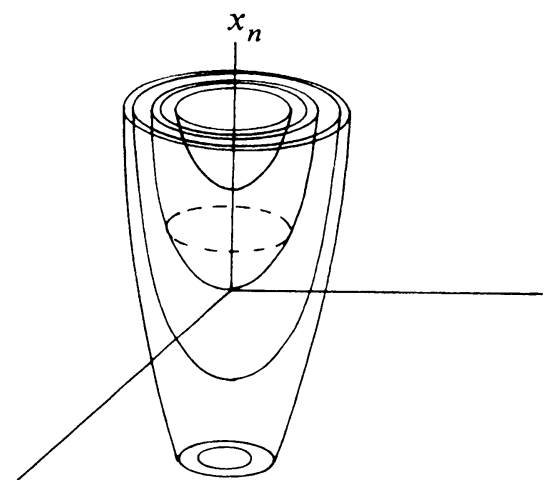

FIGURE 1

Two codimension 1 foliations of $M, \mathcal{F}$ and $\mathcal{G}$, are integrably homotopic if there exists a foliation with boundary, $\tilde{\mathscr{F}}$, of $M \times I$ such that (i) $\tilde{\mathscr{F}} \cap\{M \times$ $\{0\}\}=\mathscr{F}$ and $\tilde{\mathscr{F}} \cap\{M \times\{1\}\}=\mathcal{G}$, and (ii) $\tilde{\mathscr{F}}$ is transverse to the trivial foliation $\{M \times\{t\}: t \in I\}$. A theorem of Milnor [8] states that integrably homotopic is equivalent to isotopic. If the transversality condition is dropped then $\mathscr{F}$ and $\mathcal{G}$ are merely concordant. Any two foliations of $T^{2}$ are concordant (Thurston [13]).

The symbol " $\approx$ " denotes diffeomorphic (e.g., $\left.M \approx S^{n-1} \times I\right) . \quad \chi(M)$ is the Euler characteristic of $M$. All manifolds are smooth and orientable. The double manifold of a manifold $M$ with boundary equals $M$ glued to a copy of $M$ along $\partial M$ via $\operatorname{id}_{\partial M}$.

Reeb stability: The following Reeb stability theorem ([10], [6]) is the most important theorem on compact leaves and their topological type.

TheOREM (G. REEB). Let $\mathcal{F}$ be a $C^{r}$ foliation on a manifold $M, r \geqslant 0$, and suppose that $\mathcal{F}$ has a compact leaf $\mathcal{E}$ with finite fundamental group. Then every neighborhood of $\mathcal{L}$ contains an invariant neighborhood $U$ with the property that each leaf $\mathcal{L}^{\prime} \subset U$ is a finite covering space of $\mathcal{L}$. 
Thus, if $\mathcal{L}$ is simply connected, $U$ has the form $\mathcal{L} \times D^{q}$ with the foliation $\{\mathcal{L} \times\{x\}: x \in D\} . \quad M$ need not be compact; the following is a strengthening in the codimension $1, M$ compact case:

COROLlaRY [10], [0]. Let $M$ be a compact manifold with a smooth codimension 1 , transversely orientable foliation $\mathcal{F}$. If $\mathcal{F}$ has a compact leaf $\mathfrak{E}$ with finite fundamental group, then $M$ is a fiber bundle over $S^{1}$ with fiber $\mathcal{L}$.

1. Morse contact. It will be shown in Theorem 1(iv) that generically points of contact between two codimension 1 foliations are points of Morse contact.

DefintTION. Assume that $\mathcal{F}$ and $\mathcal{G}$ are codimension $k$ foliations of $M$ and that $f: U \rightarrow \mathbf{R}^{k}$ defines $\mathscr{F}$ locally near $x_{0}$. Assume $x_{0} \in \mathcal{L} \in \mathcal{G}$. Then $\mathscr{F}$ has Morse contact with $\mathcal{G}$ at $x_{0}$ if $G=\left.f\right|_{\mathfrak{L}}$ has a critical point at $x_{0}$ and the following $n \times k n$ matrix of second partial derivatives has maximal rank:

$$
\left(\begin{array}{llll}
\frac{\partial^{2} G_{1}}{\partial x_{i} \partial x_{j}} & \frac{\partial^{2} G_{2}}{\partial x_{i} \partial x_{j}} & \cdots & \frac{\partial^{2} G_{k}}{\partial x_{i} \partial x_{j}}
\end{array}\right) .
$$

Here the $G_{i}$ are the coordinate functions of $G$ and the $\left\{x_{i}\right\}$ are local coordinates on $U$. If all points of $\operatorname{Con}(\mathscr{F}, \mathcal{G})$ are Morse points then $\mathscr{F}$ has Morse contact with $\mathcal{G}$.

If $k=1$, then $G$ has a Morse critical point at $x_{0}$. If this critical point is index $p$ or index $(n-p)$, then $\mathcal{F}$ has index $p$ Morse contact with $\mathcal{G}$ at $x_{0}$ : Morse contact is intrinsic and does not depend on the choice of $f$.

ExAMPLES. (1) $\mathscr{F}=0$-sloped foliation of $T^{2}, \mathcal{G}$ is a snaking isotope of $\mathscr{F}$ (Figure 2). All contact is index 0 .

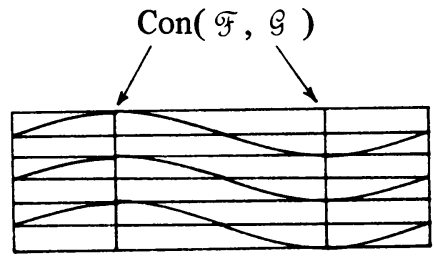

FIGURE 2

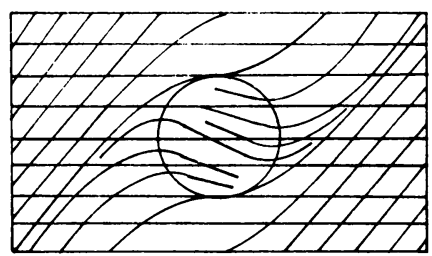

FIGURE 3

(2) $\mathscr{F}=$ the 0 -sloped foliation of $T^{2}, \mathcal{G}$ is an isotope of the 1-sloped foliation. $\operatorname{Con}(\mathscr{F}, \mathcal{G})$ is a circle. (Figure 3.) Two contact points are not Morse. Such points will be called non-Morse points. There is no foliation near $\mathcal{G}$ which has Morse contact with $\mathscr{F}$.

(3) $\mathscr{F}$ foliates $\mathbf{R}^{3}$ and is defined $f(x, y, z)=z . \mathcal{G}$ is a 1-parameter family of parabolas, defined by $g(x, y, z)=x^{2}+y^{2}-z^{2}$. (Figure 4.) Points on the $z$-axis are index 0 Morse contact points. 


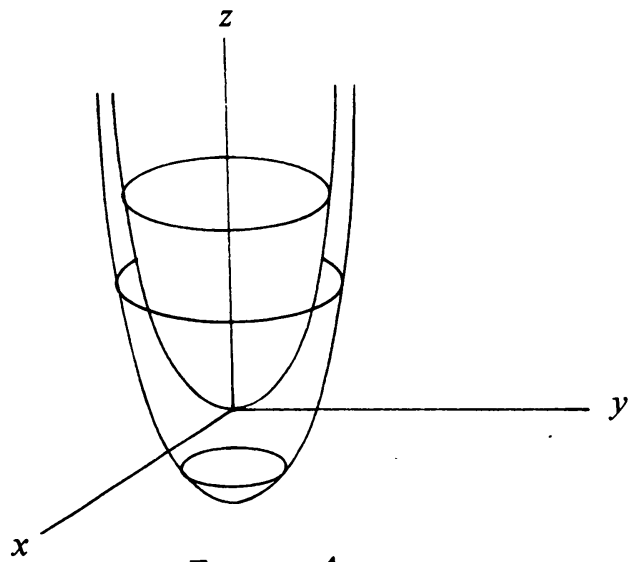

FIGURE 4

Codimension 1. When both foliations are codimension 1 , a generalization of the "Lemma of Morse" (Milnor [9]) can be proved.

Morse Contact Lemma. Assume two codimension 1 foliations $\mathcal{F}$ and $\mathcal{G}$ are locally defined on $U$ by $f$ and $g$. Assume $\mathcal{F}$ and $\mathcal{G}$ have Morse contact at $p \in U$. Then on a smaller neighborhood $V \subset U$ of $p$, there exist smooth coordinates $\left(x_{1}, \ldots, x_{n}\right)$ such that $p=(0, \ldots, 0), f=x_{n}$, and

$$
g=x_{n}-\left(x_{1}^{2}+x_{2}^{2}+\cdots+x_{\lambda}^{2}\right)+\left(x_{\lambda-1}^{2}+\cdots+x_{n-1}^{2}\right) \text {. }
$$

Proof. First we show that $\operatorname{Con}(\mathscr{F}, \mathcal{G}) \pitchfork \mathcal{F}$ at $x_{0}$. We may assume that there exist smooth coordinates $\left(x_{1}, \ldots, x_{n}\right)$ on $U$ such that $f=x_{n}$ and $p=(0, \ldots, 0)$.

$\mathscr{F}$ and $\mathcal{G}$ being codimension 1 , their plane fields $\sigma_{1}=\sigma_{\mathscr{G}}$ and $\sigma_{2}=\sigma_{\mathcal{G}}$ may be considered as maps into $\mathbf{P}^{n-1}$. In homogeneous coordinates

$$
\sigma_{1}=\left[\frac{\partial f}{\partial x_{1}}, \ldots, \frac{\partial f}{\partial x_{n}}\right]=[0,0, \ldots, 0,1] \text { and } \sigma_{2}=\left[\frac{\partial g}{\partial x_{1}}, \ldots, \frac{\partial g}{\partial x_{n}}\right] \text {. }
$$

Since $\sigma_{2}$ is nonsingular we may assume that $\partial g / \partial x_{n}$ is nonzero. This allows us to change to inhomogeneous coordinates by dividing through by the $n$ th-coordinate, then projecting onto the first $(n-1)$-coordinates. Then

$$
\sigma_{1}=[0, \ldots, 0] \text { and } \sigma_{2}=\left[\frac{\partial g / \partial x_{1}}{\partial g / \partial x_{n}}, \ldots, \frac{\partial g / \partial x_{n-1}}{\partial g / \partial x_{n}}\right]
$$

and are considered as maps into $\mathbf{R}^{n-1}$.

Restrict $\sigma_{1}$ and $\sigma_{2}$ to $U \cap \mathcal{E}$ where $p \in \mathcal{E} \in \mathcal{F}$. Now assume there exists nonzero $v_{0}=\sum_{j=1}^{n-1} a_{j} \partial / \partial x_{j} \in T_{p} \operatorname{Con} \cap T_{p} \mathcal{L}$. Two codimension 1 foliations have contact precisely where they are tangent. So $\operatorname{Con}(\mathscr{F}, \mathcal{G})=\{x \in M$ : $\left.\left(\sigma_{1}-\sigma_{2}\right)(x)=0\right\}$. If $v_{0} \in T_{p}$ Con then $v_{0}\left(\sigma_{1}-\sigma_{2}\right)=0$. But in inhomogeneous coordinates 


$$
\begin{aligned}
v_{0}\left(\sigma_{1}-\sigma_{2}\right)(p) & =\left(\sum a_{j} \frac{\partial}{\partial x_{j}}\left(-\frac{\partial g / \partial x_{1}}{\partial g / \partial x_{n}}\right), \ldots, \sum a_{j} \frac{\partial}{\partial x_{j}}\left(-\frac{\partial g / \partial x_{n-1}}{\partial g / \partial x_{n}}\right)\right)_{p} \\
& =\frac{1}{\partial g / \partial x_{n}}\left(\begin{array}{c}
\text { Hessian of } g \\
\text { at } p
\end{array}\right) \cdot v_{0} \neq 0 \text { iff } g \text { is Morse on } U \cap \mathcal{L} .
\end{aligned}
$$

So no such $v_{0}$ exists.

It follows from Theorem $1(i)$ (independently) that $\operatorname{Con}(\mathscr{F}, \mathcal{G})$ is a finite union of 1-dimensional submanifolds. From the above argument, $\operatorname{Con}(\mathscr{F}, \mathcal{G})$ is transverse to $\mathcal{L}$ at $p$. So there exist coordinates again called $\left(x_{1}, \ldots, x_{n}\right)$ such that $f=x_{n}$ and such that $\operatorname{Con}(\mathscr{F}, \mathcal{G}) \cap U$ occupies the $x_{n}$-axis. Since $\left.g\right|_{\mathfrak{L}}$ is Morse at $p$, there exists a foliation neighborhood $N$ about $\mathcal{E}$ such that for all $\mathcal{L} \in N,\left.g\right|_{\mathfrak{L}}$ is Morse at $\mathcal{L} \cap \operatorname{Con}(\mathscr{F}, \mathcal{G})$. The remainder of the proof mimics Milnor's proof of the Lemma of Morse considering instead of a single Morse function $f$, a one-parameter family of Morse functions $f_{\mu}$ each with Morse critical points on the $x_{n}$-axis. Q.E.D.

Codimension $k$. Assume two codimension $k$ foliations $\mathcal{F}$ and $\mathcal{G}$ are defined locally by $f, g: U \rightarrow \mathbf{R}^{k}$ where $f=\left(f_{1}, \ldots, f_{k}\right)$ and $g=\left(g_{1}, \ldots, g_{k}\right)$.

Conjecture (Codimension $k$ Morse Contact Lemma). If $p$ is a point of Morse contact between $\mathcal{F}$ and $\mathcal{G}$ then there exist coordinates $\left(x_{1}, \ldots, x_{n}\right)$ near $p$ such that $f_{1}=x_{n}, f_{2}=x_{n-1}, \ldots, f_{k}=x_{n-k-1}$ and

$$
g_{j}=x_{n-j+1}-G_{j}\left(x_{1}, \ldots, x_{n-j-1}\right) \text { for } 1<j<k \text {. }
$$

As indicated by Smale [11], one cannot expect each of these $G_{j}$ to be Morse functions. If $p$ is also required to be a Pareto optimal point for the set of functions $\left\{\left.g_{1}\right|_{\mathfrak{L}}, \ldots,\left.g_{k}\right|_{\mathfrak{L}}\right\} \quad(p \in \mathcal{L} \in \mathscr{F})$, then perhaps these $G_{j}$ may be Morse.

2. Generic contact. Isotransversability is not a dense property, nor is it always possible to perturb a foliation so that it has Morse contact with a fixed foliation. Generally, two foliations have as contact set the finite union of submanifold complexes (see Hirsch [3]).

Definition. Submanifolds $N_{0}, \ldots, N_{q} \subset M$ form a submanifold complex of dimension $q$ if (i) $N_{0}$ is closed and $\bar{N}_{i+1} \backslash N_{i+1} \subset N_{0} \cup \cdots \cup N_{i}$; (ii) $N_{q} \neq$ $\varnothing$; (iii) $\operatorname{dim} N_{i-1}<\operatorname{dim} N_{i}$; (iv) let $0 \leqslant i, j \leqslant q, d=\operatorname{dim} N_{i}$; then if a sequence $\left\{x_{n}\right\}$ in $N_{j}$ converges to $y$ in $N_{i}$, there is a sequence of $d$-planes $E_{n} \subset T_{x_{n}} N_{j}$ converging to $T_{y} N_{i}$.

This last condition assures that in the generic case a submanifold and a submanifold complex intersect transversally (i.e., the submanifold is transverse to each submanifold of the complex). An example of a 2-dimensional submanifold complex of $\mathbf{R}^{3}$ is the "sphere with lips". (Figure 5.) 


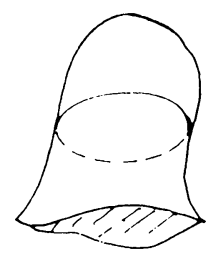

FIGURE 5

We now define two special submanifold complexes, $Q_{\sigma}$ and $\mathscr{G}_{\sigma}$, of the Grassmannian bundle associated to an $(n-k)$-plane field $\sigma$.

$$
G_{n, n-k} \hookrightarrow \begin{gathered}
G B_{n, n-k} \\
\sigma \uparrow \downarrow \\
M
\end{gathered}
$$

For $k \leqslant n / 2, \mathbb{Q}_{\sigma}=\cup_{x \in M} A_{x}$ where

$$
A_{x}=\left\{W \in G_{n, n-k}(x): W \text { is not transverse to } \sigma(x)\right\} .
$$

For $k \geqslant n / 2, \mathscr{B}_{\sigma}=\cup_{x \in M} B_{x}$ where

$$
B_{x}=\left\{W \in G_{n, n-k}(x): \operatorname{dim}(W \oplus \sigma(x))<2(n-k)\right\} .
$$

These are submanifold complexes because, for each $x, A_{x}$ and $B_{x}$ are naturally diffeomorphic to the following spaces of linear maps:

$$
\begin{aligned}
& A_{x} \approx\left\{\phi \in L\left(V^{n-k}, V^{k}\right): \phi \text { has rank }<k-1\right\}, \\
& B_{x} \approx\left\{\phi \in L\left(V^{n-k}, V^{k}\right): \phi \text { is not injective }\right\},
\end{aligned}
$$

where $V^{n-k}$ is an $(n-k)$-dimensional subspace of an $n$-dimensional vector space and where by $V^{k}$ we mean $V^{n-k^{\perp}}$. Each $A_{x}$ is a union of submanifolds, $A_{x}^{s} \approx\{\phi: \operatorname{rank} \phi=s\}, 0 \leqslant s \leqslant k-1$. Similarly for each $B_{x}$. It follows then from Hirsch [3] that $A_{x}$ is a submanifold complex of dimension $(k-1)(n-$ $k+1)$. Thus $\mathbb{Q}_{\sigma}$ is a $[(k-1)(n-k+1)+n]$-submanifold complex. Similarly $\mathscr{B}_{\sigma}$ is $[(n-k-1)(k+1)+n]$-dimensional.

Theorem 1 (Generic Contact). Fix $\mathscr{F}_{0} \in \mathrm{Fol}_{k}^{\infty}(M), M$ a smooth compact $n$-manifold $\left(\sigma_{0}=\sigma_{\mathscr{F}_{0}}\right)$.

(i) For $k \leqslant n / 2$ let $\mathscr{R}=\mathscr{R}\left(\mathscr{F}_{0}\right)=\left\{\mathscr{F} \in \operatorname{Fol}_{k}(M)\right.$ : $\left.\sigma_{\mathscr{F}} \pitchfork \mathbb{Q}_{\sigma_{0}}\right\}$. Then

(a) $\Re$ is open and dense in $\mathrm{Fol}_{k}^{\infty}(M)$, and

(b) for all $\mathscr{F} \in \Re, \operatorname{Con}\left(\mathscr{F}, \mathscr{F}_{0}\right)$ is the finite union of smooth $(2 k-1)$ dimensional submanifold complexes.

(ii) For $k \geqslant n / 2$, let $\mathscr{R}=\mathscr{R}\left(\mathscr{F}_{0}\right)=\left\{\mathscr{F} \in \mathrm{Fol}_{k}^{\infty}(M)\right.$ : $\left.\sigma_{\mathscr{F}} \pitchfork \mathscr{B}_{\sigma_{0}}\right\}$. Then

(a) $\Re$ is open and dense in $\mathrm{Fol}_{k}^{\infty}(M)$, and

(b) $\operatorname{Con}\left(\mathscr{F}_{,} \mathscr{F}_{0}\right)$ is the finite union of smooth $[2(n-k)-1]$-dimensional submanifold complexes. 
(iii) For all $\mathcal{F}$ and $\varepsilon>0$, there exists a smooth isotopy $h_{t}: M \hookleftarrow, C^{\infty} \varepsilon$-near to the identity such that $h_{1}(\mathscr{F}) \in \Re$.

(iv) When $k=1$ there exists an open and dense subset $\Re_{0}$ of $\Re\left(\mathscr{F}_{0}\right)$ such that for all $\mathscr{F} \in \Re_{0}$, all but a finite number of points of $\operatorname{Con}\left(\mathscr{F}, \mathscr{F}_{0}\right)$ are Morse. Furthermore, there exists a $C^{\infty}$-small isotopy which carries any arbitrary foliation to an element of $\Re_{0}$.

Definition. Two codimension $k$ foliations $\mathscr{F}$ and $\mathcal{G}$ have generic contact $(k>1)$ if $\mathscr{F} \in \Re(\mathcal{S})$. If $k=1$, we require that $\mathscr{F} \in \Re_{0}(\mathcal{S})$.

The proof of this theorem is complicated by the fact that perturbations of $\sigma_{\mathscr{F}}$ in $G \mathscr{B}_{n, n-k}$ must pass through integrable plane fields. The key lemma which assures that this sort of perturbation is possible is due to Lawson [7]:

LEMMA 2. Let $U, M$ and $\tilde{M}$ be smooth manifolds and $S \subset \tilde{M}$ a smooth submanifold; and let $F: U \times M \rightarrow \tilde{M} \supset S$ be a smooth map. If $F$ is transverse to $S$, then the mapping $F_{u}: M \rightarrow \tilde{M}$ given by $F_{u}(x)=F(u, x)$ is also transverse to $S$ for a.a. $u \in U$.

This lemma is applied in our case as follows:

LEMMA 2'. Let $N_{1}$ be a submanifold and $N_{2}$ be a submanifold complex of $G_{n, n-k} \times \mathbf{R}^{n}$. Then for a.a. $g=\left(g_{1}, g_{2}\right) \in \mathcal{O}_{n} \times E^{n}$ (the orthogonal and Euclidean groups), $g\left(N_{1}\right) \pitchfork N_{2}$.

Proof. First, Lemma 2 may be modified to allow $S$ to be a submanifold complex; by Hirsch [3], it is an open and dense property that a submanifold and a submanifold complex meet transversally (or cleanly).

Next, let $\tilde{M}=G_{n, n-k} \times \mathbf{R}^{n}$. Define $\mu:\left(\Theta_{n} \times E^{n}\right) \times N_{1} \rightarrow \tilde{M} \supset N_{2}$ by $\mu$ : $(g, x) \mapsto g(x)$. The $\left(\theta_{n} \times E^{n}\right)$-action on $G_{n, n-k} \times \mathbf{R}^{n}$ is transitive; thus $\mu$ is a submersion. Trivially, $\mu \pitchfork N_{2}$. Then, for a.a. $g, \mu_{g}: N_{1} \rightarrow M$ is transverse to $N_{2}$ (or clean to $N_{2}$ ) where $\mu_{g}=\mu(g, x)=g(x)$. Q.E.D.

Proof of THEOREM 1. That $\Re$ is open for all $k$ is a consequence of transversality theory; we now show density. Let $\left\{V_{i} \subset U_{i}\right\}$ be a finite collection of open balls of radii $r / 2$ and $r$, respectively, which cover $M$. Because each $V_{i}$ is diffeomorphic to $\mathbf{R}^{n}$, the action $g \in \mathcal{O}_{n} \times E^{n}$ of Lemma $2^{\prime}$ naturally induces an action on the Grassmannian bundle over $V_{i}$ :

$$
\begin{aligned}
G_{n, n-k} \hookrightarrow \quad & G B_{n, n-k} \approx G_{n, n-k} \times V_{i} \\
& \left.\uparrow \downarrow \sigma\right|_{V_{i}},\left.\sigma_{0}\right|_{V_{i}} \\
& V_{i}
\end{aligned}
$$

Let $N_{1}=\sigma\left(V_{i}\right)=\sigma_{\sigma y}\left(V_{i}\right)$ and let $N_{2}=\mathscr{Q}_{\sigma_{0}}$ when $k \leqslant n / 2$ or let $N_{2}=\mathscr{B}_{\sigma_{0}}$ when $k \geqslant n / 2$. By Lemma 2', for a.a. $g \in \Theta_{n} \times E^{n}, g\left(N_{1}\right) \pitchfork N_{2}$ or $g\left(N_{1}\right)$ is clean to $N_{2}$. This implies density on $V_{i}$.

Furthermore, for arbitrarily small $g \in O_{n} \times E^{n}$, there exists an arbitrarily 
small diffeomorphism $\tilde{g}$ on $V_{i}$ such that $g\left(N_{1}\right)=\sigma_{\tilde{g}(\mathfrak{g})}\left(V_{i}\right)$. Such $\tilde{g}$ are isotopic to the identity. (This implies (iii) on $V_{i}$.) Let $h_{t}^{i}: V_{i} \circlearrowleft$ be this isotopy. Now $\left.\tilde{g}\right|_{V_{t}}$ and $h_{t}^{i}$ are extended via a bump function to the rest of $M$, both equaling the identity outside of $U_{i}$. (On $U_{i} \backslash V_{i}$ they are bump-averaged with the identity.) This is possible because each is very small.

Next on $V_{i+1}$ an even smaller isotopy, $\tilde{h}_{t}^{i+1}$, is used which does not disturb the transversality on $V_{i}$ and which satisfies the same transversality requirements on $V_{i+1}$. Again this $\tilde{h}_{t}^{i+1}$ is extended to all of $M$. Now let $h_{t}^{i+1}=$ $\tilde{h}_{t}^{i+1} \circ h_{t}^{i}$. In this manner an isotopy is constructed which satisfies (iii). This arbitrarily small isotopy pushes $\mathscr{F}$ into $R$.

Showing (i)(b).

$$
\operatorname{Con}\left(\mathscr{F}, \mathscr{F}_{0}\right)=\left\{x \in M: \mathbb{Q}_{\sigma_{0}}(x) \cap \sigma_{\mathscr{F}}(x) \neq \varnothing\right\}=\sigma_{\mathscr{F}}^{-1}\left\{\mathbb{Q}_{\sigma_{0}} \cap \sigma_{\mathscr{F}}(M)\right\}
$$

Since $\sigma_{\mathscr{F}}$ is diffeomorphic onto its image, $\operatorname{dim} \operatorname{Con}\left(\mathscr{F}, \mathscr{F}_{0}\right)=\operatorname{dim} \mathbb{Q}_{\sigma_{0}} \cap$ $\sigma_{\mathscr{F}}(M)$. For $\mathscr{F} \in \Re$,

$$
\begin{aligned}
\operatorname{dim} Q_{\sigma_{0}}+\operatorname{dim} & \sigma_{\mathscr{F}}(M)-\operatorname{dim} G B_{n, n-k} \\
& =[(k-1)(n-k+1)+n]+[n]-[(n-k) k+n] \\
& =2 k-1 .
\end{aligned}
$$

Showing (ii)(b). For $\mathscr{F} \in \Re$,

$$
\begin{aligned}
\operatorname{dim} \operatorname{Con}\left(\mathscr{F}, \mathscr{F}_{0}\right) & =\operatorname{dim} \mathscr{B}_{\sigma_{0}}+\operatorname{dim} \sigma_{\mathscr{F}}(M)-\operatorname{dim} G B_{n, n-k} \\
& =[(n-k-1)(k+1)+n]+[n]-[(n-k) k+n] \\
& =2(n-k)-1 .
\end{aligned}
$$

Showing (iv) (All foliations are codimension 1). Let $\mathcal{G}_{0} \in \mathcal{R}\left(\mathscr{F}_{0}\right)$. We show there exists a $C^{\infty}$-small isotopy which carries $\mathcal{G}_{0}$ through $\Re\left(\mathscr{F}_{0}\right)$ to an element $\mathcal{G}$ of $\Re_{0}$. First, $\operatorname{Con}\left(\mathscr{F}_{0}, \mathcal{G}_{0}\right)$, being compact, may be covered by finitely many nested triplets of $\mathscr{F}_{0}$-boxes, $U_{i} \subset V_{i} \subset W_{i}, i=0, \ldots, N$. We assume $\operatorname{Con}\left(\mathscr{F}_{0}, \mathcal{G}_{0}\right) \subset \cup_{i=0}^{N}$ Int $U_{i}$ and that the ratios of radii of these boxes is fixed for all $i, \frac{3}{4}: \frac{7}{8}: 1$. (Figure 6.) These triplets are chosen so that (a) any $\mathcal{G}_{0}$-leaf which passes through a point $V_{i}$ is (in $W_{i}$ ) the graph of a map $g_{0}: B^{n-1} \rightarrow$ $(-1,1)$ where $\left\|D g_{0}\right\| \ll 1$, and (b) the union of the $\mathcal{G}_{0}$-leaves (in $W_{i}$ ) which pass through $V_{i} \cap(0 \times[-1,1])$ contains a neighborhood of $U_{i}$. Such triplets do exist since on Con, $\mathscr{F}_{0}$ and $\mathcal{G}_{0}$ are tangent so that by choosing these triplets very small, $g_{0}$ will have as small a derivative as need be. That the ratios of these boxes is kept constant assures that a finite number of such triplets will cover $\operatorname{Con}\left(\mathscr{F}_{0}, \mathcal{G}_{0}\right)$ in the sense that a finite number of the $U_{i}$ are a covering. 


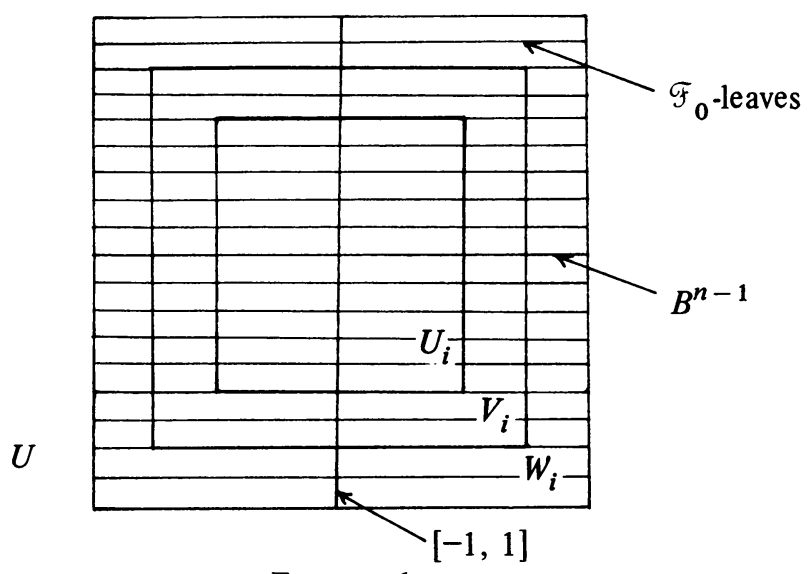

FIgURE 6

Consider $U_{0} \subset V_{0} \subset W_{0}$. Let $A=V_{0} \cap(0 \times[-1,1])=0 \times\left[-\frac{7}{8}, \frac{7}{8}\right] . g_{0}$ $=g_{0}(x, \alpha), x \in B^{n-1}$ and $\alpha \in A$ so that the $g_{0}$-leaves are $\mathcal{G}_{0}$ restricted to $W_{0}$. We apply jet-transversality as follows: Let

$$
Y=B^{n-1} \times A \times L\left(\mathbf{R}^{n-1}, \mathbf{R}\right) \times L_{s}^{2}\left(\mathbf{R}^{n-1}, \mathbf{R}\right) .
$$

This last factor is the space of bilinear maps: $\mathbf{R}^{n-1} \times \mathbf{R}^{n-1} \rightarrow \mathbf{R}$. Let $X=$ $\{(x, \alpha, T, S) \in Y: T=0$ and $\operatorname{det} S=0\}$. Then $X$ is a submanifold complex of $Y$ with codimension $n$; the codimension of $\{T=0\}$ in $L\left(\mathbf{R}^{n-1}, \mathbf{R}\right) \approx \mathbf{R}^{n-1}$ is $n-1$ and the codimension of $\{\operatorname{det} S=0\}$ is 1 in $L_{s}^{2}\left(R^{n-1}, \mathbf{R}\right)$.

Jet-transversality implies now that we can $C^{\infty}$-approximate $g_{0}$ by $g$ so that the jet map Jg: $B^{n-1} \times A \rightarrow Y \supset X$ given by

$$
J g:(x, \alpha) \mapsto\left(x, \alpha, d_{x} g_{0}(\cdot ; \alpha), d_{x}^{2} q_{0}(\cdot ; \alpha)\right)
$$

is transverse to $X$. In particular $(J g)^{-1}(X)$ has codimension $n$; hence it is finite. Besides, by transversality $(J g)^{-1}(X)$ does not change topological type under subsequent small perturbations of $g$. Furthermore, the points of $(J g)^{-1}(X) \cap U_{i}$ are precisely the set of non-Morse contact points in $U_{i}$.

Now we observe there exists a $C^{\infty}$-small isotopy with support in $W_{0}$ which vertically pushes the $\left.\mathcal{G}_{0}\right|_{U_{0}}$-leaves to the $\left.g\right|_{U_{0}}$-leaves. Thus on $U_{0}$, we have our result for a new global foliation $\mathcal{G}_{1}$, isotopic to $\mathcal{G}_{0}$ by a small isotopy. Properties of our triplets, (a) and (b), being open, continue to hold for our new $\mathcal{G}_{1}$. Openness or stability of jet-transversality assures that we may now go on to the next triplet of boxes, perturbing $\mathcal{G}_{0}$ on them without disturbing our result on $U_{0}$. Continuing, the desired $\mathcal{G} \in \Re_{0}$ is attained.

Corollary. Let $T=T_{\mathscr{F}_{0}}=\left\{\mathscr{F} \in \mathrm{Fol}_{k}^{\infty}(M): \sigma_{\mathscr{F}} \pitchfork \sigma_{\mathscr{F}_{0}}\right\}$. Then

(a) $T$ is open and dense in $\operatorname{Fol}(M)$, and

(b) For all $\mathscr{F} \in T_{\mathscr{F}_{0}}, \operatorname{Tan}\left(\mathscr{F}_{,}, \mathscr{F}_{0}\right)$ is the finite union of smooth $[n-k(n-$ $k)$-submanifolds. (When $[n-k(n-k)]<0$ Tan is empty.) 
Proof. For $\mathscr{F} \in T_{\mathscr{F}_{0}}$,

$$
\operatorname{dim}\left(\sigma_{0}(M) \cap \sigma(M)\right)=2 n-[(n-k)(n)+n]=n-k(n-k) .
$$

The rest of the proof follows the above proof of Theorem 1.

REMARKs. (i) Suppose it is known that $\operatorname{Con}(\mathscr{F}, \mathcal{G})$ has dimension less than $2 k-1(k \leqslant n / 2)$. One may not conclude that there exists a small isotopy such that $\operatorname{Con}\left(\mathscr{F}, h_{1}(\mathcal{G})\right)=\varnothing$. (Two spheres in 3-space which intersect in a point may always be perturbed apart.) The following example is due to Hirsch [4]: Let $X$ be a nonvanishing vector field on $S^{5} \cdot(\operatorname{Im} X)^{\perp} \subset T S^{5}$ is a 9-manifold. Bump $X$ to $Y$ so that $Y$ has one singularity. The projections of $Y$ and the zero section into $(\operatorname{Im} X)^{\perp}$ are two 5-submanifolds which intersect in a point. $S^{5}$ has at most one linearly independent nonvanishing vector field [12]-so the two 5-submanifolds cannot be perturbed apart.

(ii) In the Franks and Robinson isotopy [1], $n=3, k=2$. Then generically $\operatorname{dim}$ Con $=1$. This is in fact the dimension of Con before their large isotopy.

(iii) Not all the intermediate lower dimensions of submanifolds of the complex Con necessarily occur. These dimensions may be calculated starting with $\operatorname{dim} A_{x}^{s}$.

\section{Domed contact.}

DEFINITIONS. (1) $\mathcal{F}$ is a foliation with centers of $M^{n}$ if about each point of $M$ there exist an open neighborhood $U$ and Morse function $f: U \rightarrow \mathbf{R}$ such that the leaves of $\mathscr{F} \cap U$ are the level surfaces of $f$ and all singularities of $f$ are either index 0 or index $n$.

(2) Two foliations $\mathcal{F}$ and $\mathcal{G}$ have domed contact if $\operatorname{Con}(\mathscr{F}, \mathcal{G}) \neq \varnothing$ and all contact is Morse of index 0 .

(3) A foliation with boundary $\tilde{\mathscr{F}}$ of $M \times I$ bridges two foliations with centers $\mathscr{F}$ and $\mathcal{G}$ if (i) $\tilde{\mathscr{F}} \cap\{M \times\{0\}\}=\mathscr{F}$ and $\tilde{\mathscr{F}} \cap\{M \times\{1\}\}=\mathcal{G}$, and (ii) $\tilde{\mathscr{F}}$ has domed contact with the trivial foliation $\{M \times\{t\}\}$.

(4) Denote $M_{t}=M \times\{t\}$.

EXAMPLE 1. $\mathcal{G}$ is the trivial foliation of $S^{2} \times I$ (consisting of concentric spheres). $\tilde{\mathscr{F}}$ is a foliation with boundary containing two Reeb sectors. The rest of $\tilde{\mathscr{F}}$ is filled out by annular leaves. $\tilde{\mathscr{F}}$ has domed contact with $\mathcal{G}$ along two radial line segments. Everywhere else the two foliations are transverse. $\tilde{\mathscr{F}} \cap$ $M_{0}$ and $\tilde{\mathscr{F}} \cap M_{1}$ are foliations with centers bridged by $\tilde{\mathscr{F}}$. Notice that if one of the Reeb sectors were reversed in orientation, this new foliation would still bridge $\tilde{\mathscr{F}} \cap M_{0}$ and $\tilde{\mathscr{F}} \cap M_{1}$; the isotopy class of the bridging foliation is not unique. (Figure 7.)

THEOREM 2 (DOMED CONTACT). Let $M^{n}$ be a compact orientable manifold with boundary, $n>3$. A proper codimension 1 foliation $\mathcal{F}$ of $M$ and a codimension 1 foliation with boundary, $\mathcal{G}$ (such that $\operatorname{Con}(\mathcal{F}, \mathcal{G})$ meets $\partial M)$ have domed 
contact if and only if

(i) $M$ is homeomorphic to $S^{n-1} \times I$,

(ii) $\mathscr{F}$ is isotopic to the trivial foliation, and

(iii) $\mathcal{G}$ is in one of five certain isotopy classes.

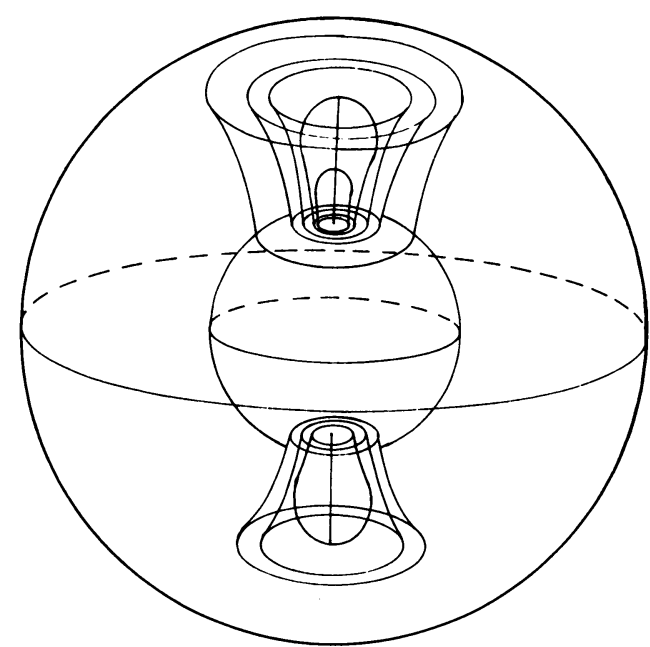

FIGURE 7

Proof. $(\Rightarrow)$ (Proof of (iii) due to Glenn Davis.)

Showing (i). Assume $\mathcal{G}$ has domed contact with $\mathcal{F}$ at $p_{0}$, an element of the boundary component $\partial_{0} M$. Then on a neighborhood of $p_{0}, U \subset \partial_{0} M$, live coordinates $\left(x_{1}, \ldots, x_{n-1}\right)$ and a defining function $f: U \rightarrow \mathbf{R}$ of $\mathcal{G} \cap U$ given by $f\left(x_{1}, \ldots, x_{n-1}\right)=x_{1}^{2}+\cdots+x_{n-1}^{2}$. Leaves near $p_{0}$ are $(n-2)$-spheres: $\mathcal{G} \cap U$ is a foliation with one center.

$M$ compact implies that such points of domed contact with $\partial_{0} M$ are finite; call them $\left\{p_{i}\right\}_{i=0}$. Near each $p_{i}$, extract a small open $(n-1)$-ball, $B_{i} \subset \partial_{0} M$, in such a way that $\mathcal{G}^{\prime}=\left\{(\mathcal{G} \cap \partial M) \backslash \cup B_{i}\right\}$ is a proper foliation of $V=\partial M$ $\backslash \cup B_{i}$.

We now show that $V \approx S^{n-2} \times I$. If $n=3, V$ is a 2-manifold with boundary the union of circles. The only such (orientable) foliatable manifold is the cylinder $S^{1} \times I$. For $n>3, V$ is an $(n-1)$-manifold with boundary the union of simply connected $(n-2)$-spheres. Let $\tilde{V}$ be the double of $V$ and $\tilde{\mathcal{G}}^{\prime}$ the double of $\mathcal{G}^{\prime}$. As a consequence of the Reeb stability theorem [10], [6], since $\tilde{\mathcal{G}}^{\prime}$ contains a compact leaf with finite fundamental group $\left(\approx S^{n-1}\right), \tilde{V}$ is a fiber bundle over $S^{1}$ with fibers $S^{n-2}$. Thus the reduced Mayer-Vietoris sequence

$$
\cdots \tilde{H}_{1}(\partial V) \rightarrow \tilde{H}_{1}(V) \oplus \tilde{H}_{1}(V) \rightarrow \tilde{H}_{1}(\tilde{V}) \rightarrow \tilde{H}_{0}(\partial V) \rightarrow 0
$$

has $\tilde{H}_{1}(\partial V) \approx 0$ and $\tilde{H}_{1}(\tilde{V}) \approx \mathbf{Z}$. Therefore $\tilde{H}_{1}(V) \oplus \tilde{H}_{1}(V) \approx 0$ since no 
other subgroup of $\mathbf{Z}$ besides $\mathbf{0}$ is the direct sum of two identical subgroups of Z. Consequently, $\tilde{H}_{0}(\partial V) \approx \mathbf{Z}$; $\partial V$ has two components. A topological argument concludes that there exist $\theta_{1}$ and $\theta_{2}$ in $S^{1}$ such that $\partial V$ is the union of fibers over $\theta_{1}$ and $\theta_{2}$. Thus there exist two copies of $V$ in $\tilde{V}$, fiber bundles over the intervals $\left[\theta_{1}, \theta_{2}\right]$ and $\left[\theta_{2}, \theta_{1}\right]$ respectively in $S^{1}$. $\left[\theta_{1}, \theta_{2}\right]$ contractible implies that $V \approx S^{n-2} \times I$.

By gluing back the $(n-1)$-balls, we conclude that $\partial_{0} M$ is homeomorphic to $S^{n-1}$, for all $n \geqslant 3$. We repeat the argument to conclude that for $n>3$, $M^{n}$ is homeomorphic to $S^{n-1} \times I$. (For $n>8$, these $(n-1)$-spheres may have exotic differentiable structures, depending on the choice of the gluing map.)

Showing (ii). $\mathscr{F}$ contains the simply connected leaf $M_{0} \approx S^{n-1}$. The Reeb stability theorem implies then that there exists a foliation neighborhood $U$ about $M_{0}$ of the form $M_{0} \times I$ and foliated by $\left\{M_{0} \times\{t\}: t \in I\right\}$ (for some interval $I$ ).

Let $g: M \rightarrow[a, b]$ be a Morse function with properties: (1) $g\left(M_{0}\right)=a$, $g\left(M_{1}\right)=b$, and (2) for all leaves $\mathcal{L} \in U, g(\mathcal{L})$ is a regular value with level surface $\mathcal{L}$. Now assume there exists $c \in(a, b)$ such that $(1)[a, c)$ are regular values of $g$, (2) for all $x \in[a, c)], g^{-1}(x)$ is a spherical leaf of $\mathscr{F}$, and (3) $c$ is the least upper bound with respect to (i) and (ii). By continuity, $g^{-1}(c)$ is a leaf of $\mathcal{F}$. So $c$ cannot be a critical value of $g$. Therefore $g^{-1}[a, c) \approx[a, c) \times$ $S^{n-1}$ and has as one of its boundaries $g^{-1}(c) \approx S^{n-1}$. But the Reeb stability theorem implies there must exist a foliation neighborhood of spherical leaves about $g^{-1}(c)$. So no such $c \in(a, b)$ exists. All the leaves of $\mathscr{F}$ are $(n-1)$ spheres; $\mathcal{F}$ is isotopic to the trivial foliation.

Showing (iii). A similar argument concludes that $\mathcal{G}_{0}=\mathcal{G} \cap M_{0}$ and $\mathcal{G}_{1}=\mathcal{G}$ $\cap M_{1}$ are both isotopic to the standard north pole-south pole foliation (with two centers) of $S^{n-1}$. We assume then that $\mathcal{G}$ bridges $\mathcal{G}_{0}$ to itself. By a preliminary isotopy we may assume that $\operatorname{Con}(\mathcal{F}, \mathcal{G})=(p \times I) \cup(q \times I)$ where $p$ and $q$ are the centers of $\mathcal{G}_{1}$. Also assume that there exist small disks $D_{p}$ and $D_{q}$ about $p$ and $q$ such that $\partial\left(D_{p} \times I\right) \cap \mathcal{G}$ and $\partial\left(D_{q} \times I\right) \cap \mathcal{G}$ are the trivial foliations of $S^{n-2} \times I$ by spheres. Each leaf of this foliation is contained in the same $t$-level of $M \times I$.

There are three possibilities depending on how the leaves of $\mathcal{G}$ pass through $p$ and $q$ (Figure 8). Recall that the Morse index is constant along $p \times I$ and $q \times I$.

Case 1. We show in this case that $\mathcal{G}$ is isotopic to a particular foliation with boundary, $\mathscr{K}_{0}$. When $n=3, \mathscr{H}_{0}$ is the spin of the foliation of the annulus shown in Figure 9. By the Morse contact lemma (and possibly by shrinking $D_{p}$ and $\left.D_{q}\right)$, we may assume that $\mathcal{G}$ and $\mathcal{F}_{0}$ agree on $\left(D_{p} \times I\right) \cup\left(D_{q} \times I\right)$.

Let $K=(M \times I) \backslash \operatorname{int}\left(D_{p} \times I\right) \backslash \operatorname{int}\left(D_{q} \times I\right)$. So $K \subset M \times I$ and inherits 


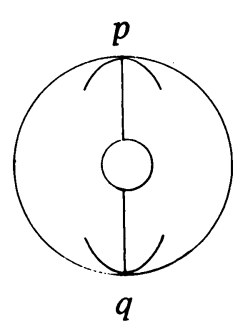

CASE 1

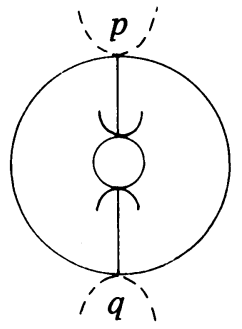

CASE 2

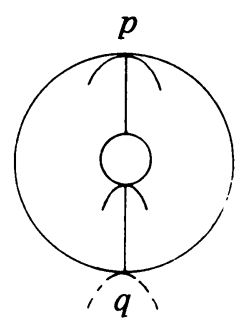

CASE 3

FIGURE 8

coordinates $(x, \tau)$. There being no contact, $\mathcal{G}$ and $\mathcal{F}_{0}$ are both transverse to $\mathscr{F}$ on $K$. Now construct a vector field $X_{\mathcal{G}}$ on $K$ which is tangent to $\mathcal{G}$ and which projects to $\partial / \partial \tau$ on $I$. Similarly for $X_{0}=X_{\mathscr{C}_{0}}$. Since $\mathcal{G}$ and $\mathcal{T}_{0}$ are Case 1, these vector fields point out of $K$ on $\partial\left(D_{p} \times I\right) \cup \partial\left(D_{q} \times I\right)$ (Figure 10). Denote by $\phi_{\mathcal{S}}$ the flow generated by $X_{\mathcal{G}}$, and by $\phi_{0}$ the flow generated by $X_{0}$.

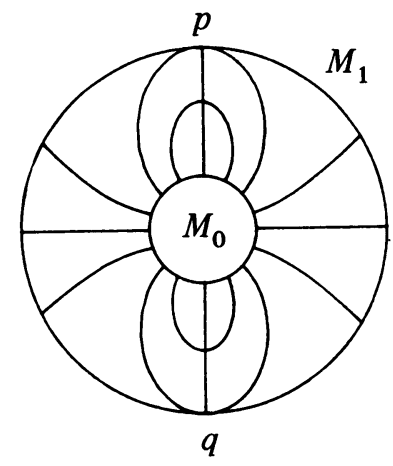

FIGURE 9

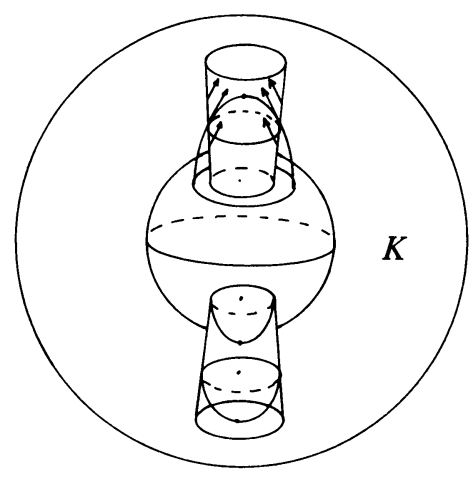

FIGURE 10

Let $N=\left[M_{1} \backslash\left(D_{p} \times\{1\}\right) \backslash\left(D_{q} \times\{1\}\right)\right] \cup\left(\partial D_{p} \times I\right) \cup\left(\partial D_{q} \times I\right)$. It is important to note that $\mathcal{G}$ and $\mathcal{H}_{0}$ agree on $N$. Define $g_{t}: K \hookleftarrow, t \in[0,1]$, as follows: If $\tau>t, g_{t}(x, \tau)=(x, \tau)$. If $\tau \leqslant t$, flow along $\phi_{S}$ from $(x, \tau)$ until either the $I$-coordinate has reached $t$ or $N$ is encountered. Then flow in negative time by $\phi_{0}$ until the $I$-coordinate is once again $\tau$. This point is defined to be $g_{t}(x, \tau)$. The reverse operation describes $g_{t}^{-1} ; \mathcal{G}$ being of Case 1 assures that this operation is well defined. It follows that $g_{t}$ is an isotopy with $g_{0}=\mathrm{id}_{K}$.

$g_{t}$ fixes $N$ for all $t$. Since $\partial\left(D_{p} \times I\right) \cup \partial\left(D_{p} \times I\right) \subseteq N$, the map $G=g_{1} \cup$ $\mathrm{id}_{D_{p} \times I \cup D_{q} \times I}$ is well defined. Then $G$ is isotopic to the identity (through $g_{t}$ ). Since $\mathcal{G}$ and $\mathcal{T}_{0}$ agree on $N, g_{1}$ and hence $G$ takes leaves of $\mathcal{G}$ to leaves of $\mathcal{F}_{0}$. (Notice that $g_{t}(t<1)$ does not necessarily take leaves to leaves since 
some pairs of points are sent up to the $t$ level but then are reversed down different leaves of $\mathcal{H}_{0}$.) Thus $\mathcal{G}$ and $\mathcal{H}_{0}$ are isotopic bridging foliations.

Case 2. A reflection of Case 1.

Case 3. In this case, we show $\mathcal{G}$ is contained in one of three isotopy classes. Again define $\phi_{\mathcal{G}}$ on $K$ (cf. Figure 11.) Let

$$
\begin{aligned}
& S_{p}=\{\text { leaf of } \mathcal{G} \text { passing through } p \times 1\} \cap\left(\partial D_{p} \times I\right), \\
& S_{q}=\{\text { leaf of } \mathcal{G} \text { passing through } q \times 0\} \cap\left(\partial D_{q} \times I\right) .
\end{aligned}
$$

So $S_{p}$ and $S_{q}$ are $(n-2)$-spheres at constant $\tau$-levels. Let $\tau_{p}=\{I$-coordinate of $S_{p}$ \}. Consider the evolution of $S_{q}$ under $\phi_{Q}$. Points flow out of $D_{q} \times I$ under $\phi_{\mathcal{G}}$, so $S_{q}$ cannot return to $\partial\left(D_{q} \times I\right)$. Since $\phi_{\mathcal{G}}$ has unit speed in the $\partial / \partial \tau$ - or $I$-direction, $S_{q}$ maintains constant $\tau$-coordinate at each forward time. Thus there are three possibilities: $S_{q}$ flows into $N$ (i) with $I$-coordinate $>\tau_{p}$, (ii) with $I$-coordinate $<\tau_{p}$, or (iii) with $I$-coordinate $=\tau_{p}$.

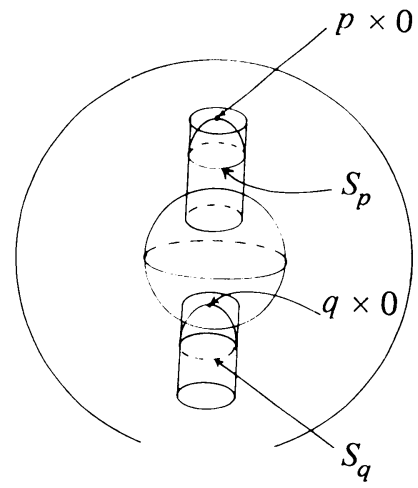

FiguRE 11

Consider case (i). If this $I$-coordinate is $<1$, then $S_{q}$ must have hit $\partial\left(D_{p} \times I\right)$ "above" $S_{p}$, say at $S_{0} . S_{0}$ is contained in a leaf of $\mathcal{G}$ which intersects $M_{1}$ near $p$. (Figure 12.) So if $D_{p}$ (and hence $S_{p}$ ) is chosen smaller this forward saturation of $S_{q}$ would not intersect $\partial\left(D_{p} \times I\right)$. We assume that $S_{q}$ hits $N \cap M_{1}$ in forward time. The picture is exaggerated as in Figure 13.

We now show that $\mathcal{G}$ is isotopic to a foliation with boundary, $\mathcal{H}_{1}$. When $n=3, \mathcal{H}_{1}$ is the spin of the following foliation of the annulus. (Figure 14.)

Let $A$ be the saturation of $\partial\left(D_{q} \times I\right)$ under $\phi_{G}$. Let $U$ be an $\varepsilon$-open neighborhood of $A$. Define $g_{t}^{\prime}: U \rightarrow K$ as follows: If $\tau \leqslant 1-t, g_{t}^{\prime}(x, \tau)=$ $(x, \tau)$. If $\tau \geqslant 1-t$ flow backwards along $\phi_{\mathcal{G}}$ until either the $I$-coordinate reaches $1-t$ or until $N$ is encountered. Then flow forward along $\phi_{\mathcal{F}_{1}}$ until the $I$-coordinate is again $\tau$. This point is $g_{t}^{\prime}(x, \tau)$. Again $g_{t}^{\prime}$ is an isotopy of $U$ with $g_{1}^{\prime}=\mathrm{id}_{U}$ which carries leaves of $\mathcal{G}$ to leaves of $\mathcal{H}_{1}$.

Now apply the isotopy extension theorem (Hirsch, [3, p. 180]) to get an 


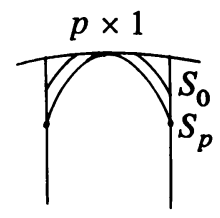

forward saturation of $S_{q}$

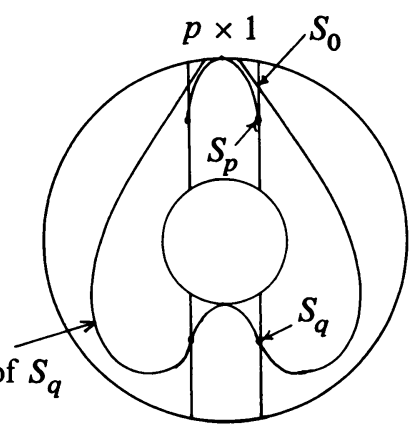

cross-section of $M \times I$

FIGURE 12

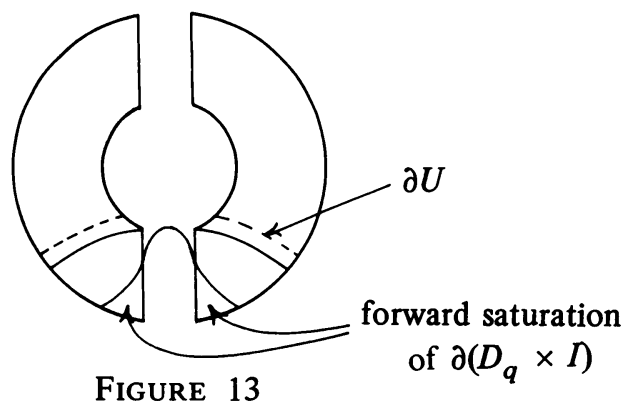

FIGURE 13

isotopy of $K$ which carries leaves of $\mathcal{G}$ contained in $A$ to leaves of $\mathcal{G}_{1}$. We now make the assumption that $\mathcal{S}$ and $\mathcal{F}_{1}$ are identical on $A$ as well as $\phi_{9}$ and $\phi_{\mathscr{C}_{1}}$. Hence the isotopy $g_{t}: K \circlearrowleft$ defined as in Case 1 is the identity on $A$. Once again $g_{1}$ is extended to $G ; \mathcal{G}$ and $\mathcal{F}_{1}$ are isotopic bridging foliations.

Case (ii) ( $S_{q}$ flows into $N$ with $I$-coordinate $\left.<\tau_{p}\right)$. In this case a similar proof shows that $\mathcal{G}$ is isotopic to a foliation $\mathcal{F C}_{2}$. For $n=3$, this is the spin of the foliation of the annulus shown in Figure 15.

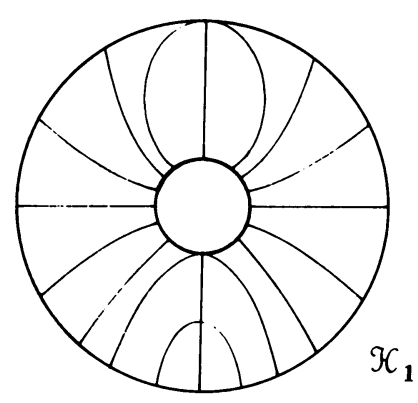

Figure 14

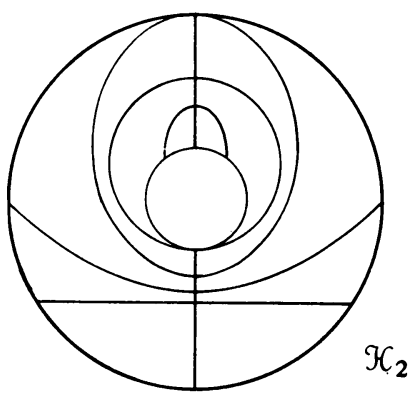

FIGURE 15

Case (iii) $\left(I\right.$-coordinate $\left.=\tau_{p}\right)$. In this case $\mathcal{G}$ is isotopic to a "degenerate" foliation $\mathcal{K}_{3}$; see Figure 16. 


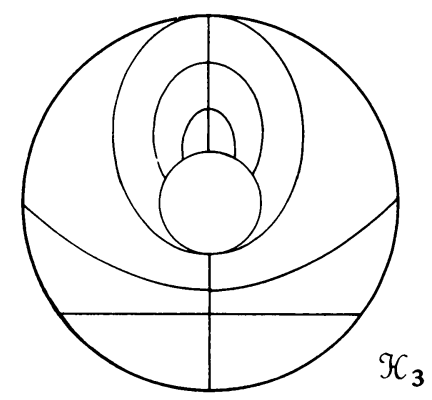

Figure 16

$(\Leftarrow)$ Knowing that $\mathcal{G}$ is isotopic to $\mathcal{K}_{0}$, a reflection of $\mathcal{K}_{0}, \mathcal{K}_{1}$, or $\mathcal{K}_{3}$ makes this implication trivial.

REMARK. If $n=3$ then (i) and (ii) follow identically. But the isotopy classes of $\mathcal{G}$ are infinite in number. This is because the Reeb stability theorem does not apply to nonsimply connected leaves, in this case, to circle leaves. These circle leaves may bound Reeb components.

\section{REFERENCES} appear).

1. J. Franks and C. Robinson, A quasi-Anosov diffeomorphism which is not Anosov (to

2. P. Hartman, Ordinary differential equations, Hartman, Baltimore, Md., 1973.

3. M. Hirsch, Differential topology, Graduate Texts in Math., no. 33, Springer-Verlag, New York, 1976.

4. Private communications.

5. B. Lawson, Lectures on the quantitative theory of foliations, Washington University Press, St. Louis, Mo., 1975.

6. __ Foliations, Bull. Amer. Math. Soc. 80 (1974), 369-418.

7. B. Lawson and F. R. Harvey, On boundaries of complex analytic varieties. I, Ann. of Math. (2) 102 (1973), 283.

8. J. Milnor, Foliations and foliated vector bundles, Princeton Univ., mimeographed notes, 1970.

9. J., 1963. , Morse theory, Ann. of Math. Studies, no. 51, Princeton Univ. Press, Princeton, N.

10. G. Reeb, Sur certains properiétés topologiques des variétés feuilletées, Actualités Sci. Indust. No. 1183, Hermann, Paris, 1952.

11. S. Smale, Global analysis and economics. I. Dynamical systems, Academic Press, New York, 1973.

12. E. Thomas, Vector fields on manifolds, Bull. Amer. Math. Soc. 75 (1969), 643-683.

13. W. Thurston, Noncobordant foliations of $S^{3}$, Bull. Amer. Math. Soc. 78 (1972), 511-514.

14. R. Walker, An index of contact on $T^{2}$; toral combinatorics, J. Combinatorics (to appear).

Department of Mathematics, University of Colorado, Boulder, Colorado 80309 